\title{
Determinación experimental de la velocidad de llama del gas de Camisea-Perú
}

\author{
Freddy J. Rojas ${ }^{(1,2) \star}$, Fernando O. Jiménez ${ }^{(1)}$ y Jorge Díaz ${ }^{(1)}$ \\ (1) Ing. Mecánica, Pontificia Universidad Católica del Perú, Av. Universitaria 1801, San Miguel, Lima-Perú. \\ (correo-e: fjrojas@pucp.edu.pe, ojimene@pucp.edu.pe, jdiaz@pucp.pe) \\ (2) Grupo de Investigación de Metodologías Aplicadas al Control Ambiental y Eficiencia Energética (GICA), Pontificia \\ Universidad Católica del Perú, San Miguel-Lima, Perú.
}

${ }^{*}$ Autor a quien debe ser dirigida la correspondencia.

Recibido Dic. 23, 2019; Aceptado Feb. 21, 2020; Versión final Abr. 18, 2020, Publicado Ago. 2020

\begin{abstract}
Resumen
El objetivo del presente trabajo de investigación ha sido determinar la velocidad de llama del gas natural de Camisea-Perú en un rango amplio de razón de equivalencia mediante un estudio experimental. Se ha implementado los cálculos matemáticos utilizando el método de la teoría térmica mediante MathCad y simulando en un mecanismo cinético actual utilizando el programa CHEMKIN. Se ha conseguido experimentalmente valores de velocidad de llama entre $5 \%$ al $10 \%$ mayor al metano $100 \%$ puro comparado con otros autores en un amplio rango de razón de equivalencia. Esto valida los resultados debido a que el gas ensayado es gas natural con $88.54 \%$ de metano y $10.32 \%$ de etano, mejorando de esta manera el banco de ensayo experimental del laboratorio de combustión y control de emisiones gaseosas. En conclusión, la metodología empleada proporciona una adecuada base de datos de velocidad de llama para el gas natural de Camisea-Perú.
\end{abstract}

Palabras clave: gas natural; gas de Camisea; velocidad de llama; Chemkin; Ilama adiabática

\section{Experimental determination of the gas flame velocity of Camisea-Peru}

\begin{abstract}
The objective of the present research study was to determine the flame speed of Camisea-Peru natural gas in a wide range of equivalence ratio through an experimental study. Mathematical calculations were implemented using the method of thermal theory in MathCad. Simulations in a current kinetic mechanism were performed using the CHEMKIN program. Values of flame velocity were $5 \%$ to $10 \%$ higher than $100 \%$ pure methane when compared to other authors in a wide range of equivalence ratio. This validated the results since the gas tested was natural gas with $88.54 \%$ methane and $10.32 \%$ ethane, thus improving the experimental test bench of the laboratory for the combustion and control of gaseous emissions. In conclusion, the methodology presented here provides an adequate database of gas flame velocity for natural gas of Camisea-Peru.
\end{abstract}

Keywords: natural gas; Camisea gas; flame speed; Chemkin; adiabatic flame 


\section{INTRODUCCIÓN}

En el Perú se está viviendo lo que es la explotación y utilización de un recurso energético relativamente nuevo para nosotros, puesto que recién se ha empezado a explotar desde la década pasada, nos referimos al Gas Natural Seco (Gas de Camisea-Perú), cuyo pronóstico de abastecimiento en nuestro país es de alrededor de 40 a 50 años. Es tanto el impacto de esta fuente de energía que actualmente cerca del $50 \%$ de energía eléctrica es producida gracias a este recurso (Tamayo et al., 2016). Además, se provee que para el 2020, un millón de peruanos ya tendrían acceso al Gas Natural Seco, desde conexiones domiciliarias, siendo un reto masificar el uso de este recurso en todo el país. Perú a su vez ha sabido adaptarse al cambio de combustible, para su propio beneficio, utilizando máquinas que puedan trabajar sin problema alguno a la intercambiabilidad de combustible, sin embargo, para un mejor uso del Gas Natural Seco, el cual cabe mencionar que es un recurso no renovable, se debe realizar mejoras en el diseño de estas máquinas con la finalidad de poder tener una mayor eficacia en su utilización.

Un factor clave para la mejora de diseños, es el parámetro de la velocidad de la llama, el cual es la velocidad con la que la llama se propaga una vez iniciado el proceso de combustión hacia los gases aún no quemados, si bien el gas natural se puede aproximar a una composición de metano $\left(\mathrm{CH}_{4}\right)$ el cual abarca en casi un $90 \%$ del total de la mezcla, no sería lo suficientemente exacto tomar valores de ello, dado a que esta propiedad es única para una mezcla determinada, y cambiaría debido a sus otros componentes que lo conforman. Una forma efectiva de hallarla es por el método experimental, donde se tiene que tener en consideración diversas condiciones de presión, temperatura de la mezcla, relación de aire-combustible, composición química de combustible. El método experimental y analítico son los utilizados en este trabajo para determinar la velocidad de la llama del Gas de Camisea-Perú.

En el año 2011, la publicación de los autores de Patricia Dirrenberger y otros realizaron estudios principalmente de los compuestos del gas natural, como lo son el metano, etano, butano, determinando la velocidad de la llama laminar, el cual consideran un parámetro importante tanto para el diseño de quemadores y predicciones de explosiones, así como un dato útil influyente para el proceso de combustión de un motor encendido por chispa (Dirrenberger et al., 2011). Además, compararon tres tipos de gases naturales, identificando cuál componente sería el más influyente en la variación de la velocidad de la llama. Para la experimentación el método a utilizar fue el de flujo térmico para estabilizar las llamas planas adiabáticas y medir las velocidades de combustión adiabática.

En los resultados y conclusiones más relevantes se puede considerar las siguientes: dentro de todos los componentes del gas natural la velocidad de llama del metano es la más baja, siendo su máximo de $38 \mathrm{~cm} / \mathrm{s}$ para una relación de equivalencia de 1.1, mientras que para el etano sucedió lo contrario pues tuvo las velocidades más altas siendo $42 \mathrm{~cm} / \mathrm{s}$ el máximo para una relación de equivalencia de 1.1 , siendo $2 \mathrm{~cm} / \mathrm{s}$ más rápido que el propano $(40 \mathrm{~cm} / \mathrm{s})$ a la misma relación de equivalencia. Las experimentaciones fueron hechas a $298 \mathrm{~K}$, presión atmosférica y con aire sintético. Los resultados concluyen que la velocidad de la llama laminar del gas compuesto parece ser determinado por el metano debido a que no hay grandes diferencias en el propano, además para los perfiles de velocidad se encuentran entre las velocidades de metano puro y etano puro, siendo más cercano al del metano. También se realizó un experimento de las mezclas metano y etano, variando su composición, así como la relación de equivalencia de 0.7 a 1.5. Donde se concluye que para todas las variaciones hasta el $50 \%$ de metano son lineales, después de este rango la velocidad de la llama suele caer pronunciadamente, cuanto más metano haya. (Dirrenberger et al., 2011).

En el año 2013, en una publicación de los autores Drew Plichta y otros presentan los resultados experimentales para determinar la velocidad de llama laminar de gas natural con mezcla de hidrogeno bajo diferentes presiones y temperaturas, esto lo hacen con el objetivo de poder determinar los efectos de la mezcla, esto debido a que en la actualidad existe una flexibilidad de combustible en motores de turbina a gas, cuyos diseñadores necesitan un dato más preciso para mejorar el diseño. Su trabajo se llevó a cabo bajo el método de la llama esférica. Utilizaron 4 experimentos cada uno con diferente tipo de mezcla, en su mayoría $\mathrm{CH}_{4}$ con diferentes porcentajes de $\mathrm{H}_{2}$, y también un experimento de una composición de gas natural $\left(81.25 \% \mathrm{CH}_{4}, 10 \% \mathrm{C}_{2} \mathrm{H}_{6}, 5 \% \mathrm{C}_{3} \mathrm{H}_{8}, 2.5 \% \mathrm{nC}_{4} \mathrm{H}_{10}\right.$ y $\left.1.25 \% \mathrm{nC}_{5} \mathrm{H}_{12}\right)$ y también se mezclaba el oxidante que generalmente es aire con He. Los resultados de los experimentos fueron comparados con el mecanismo cinético de Aramco Mech 1.0. Las conclusiones fueron las siguientes, las velocidades de mezcla mostraron comportamiento no lineal, a diferencia de cada uno de los constituyentes de combustible puro. La dilución de helio a presiones elevadas tuvo un efecto positivo en mantener la llama laminar más estable, facilitando su medida (Plichta et al., 2013).

En el año 2013, César Cardona y otros, realizaron un trabajo sobre la determinación de la velocidad de la llama laminar de gas natural con mezclas de syngas/aire, debiéndose a sus necesidades de su nación, Colombia, donde su reserva de gas natural, representa el $25 \%$ de su consumo de energía, ellos buscan 
mejoras en sus diseños de equipos, es así que tienen como objetivo obtener una viabilidad en mezclas de gas natural e hidrógeno con gas síntesis, el cual es otro recurso energético que poseen en su país, esto bajo variaciones de equivalencia pobre y rica. Su procedimiento fue realizado a través del método del ángulo, utilizando las imágenes de Schlieren. También utilizaron mecanismos cinéticos para su metodología numérica para poder comparar los resultados. Las conclusiones de estos experimentos fueron que la presencia de $\mathrm{CO}$ y $\mathrm{H}_{2}$ en la mezcla de combustible produce radicales $\mathrm{H}$, por consecuente los radicales $\mathrm{OH}$ aumenta, es decir el aumento neto en la concentración de $\mathrm{OH}$ y $\mathrm{H}$ en presencia de $\mathrm{CO}$ y $\mathrm{H}_{2}$ aumenta la tasa de consumo de metano, es decir la adición de $\mathrm{H}_{2}$ y $\mathrm{CO}_{\text {a CH}}$ aumenta la reactividad del metano (Cardona et al., 2013).

En el año 2015, los autores B. Zhang y otros provenientes de China, realizaron un estudio experimental de la mezcla de gas natural, monóxido de carbono y aire. Definen que la velocidad de la llama es un parámetro importante el cual posee información de la reactividad, difusividad y exotermicidad, cuyo valor puede afectar a la propensión de una llama a flashblack y blowoff. Además, analizan opciones para una mejora de la eficiencia de la combustión con un fin de mejora ambiental, analizando específicamente los gases de entrada a una turbina de gas.

El método utilizado fue el de la llama Bunsen, donde el experimento se realizó con gas natural $\left(95 \% \mathrm{CH}_{4}\right.$, $0.807 \% \mathrm{C}_{2} \mathrm{H}_{6}, \quad 0.102 \% \mathrm{C}_{8} \mathrm{H}_{3}, 2.980 \% \mathrm{CO}_{2}$ ) y $\mathrm{CO}$, a diferentes relaciones de equivalencia. Los resultados obtenidos fueron que la tendencia cambiante de la velocidad de la llama no es monótona con el aumento de la fracción volumétrica de gas natural en la mezcla. Las máximas velocidades de mezclas gas natural con monóxido de carbono se alcanza cuando hay un $8 \%$ de gas natural en la mezcla. Esta tendencia no monótona del cambio de velocidad de la llama laminar se debería al efecto de la tasa de reacción y la densidad de producción de calor, pues representa transiciones de la cinética dominante (Zhang et al., 2015).

En el año 2017, R. Amirante y otros recopilaron las mediciones de las velocidades de llama laminar obtenidas por varios trabajadores con el objetivo de desarrollar correlaciones empíricas más precisas para las velocidades de llama laminar en función de la relación de equivalencia y la temperatura y presión de la mezcla no quemada en un amplio rango de condiciones de operación. El propósito es proporcionar expresiones simples y factibles para modelar la velocidad de llama laminar de combustibles prácticos utilizados en motores de encendido por chispa. Se consideran compuestos puros, como metano y propano y mezclas binarias de metano / etano y metano / propano, así como combustibles más complejos, incluidos el gas natural y la gasolina (Amirante, Distaso, Tamburrano, y Reitz, 2017).

En el 2016, Y. Hong y otros investigaron los efectos de la adición de hidrógeno en las velocidades de llama laminar de metano, etano y propano. En este trabajo, se utilizó un método de llama plana para medir la velocidad de la llama laminar en una configuración de contraflujo combinada con un sistema de velocimetría de imagen de partículas (PIV). Los resultados indican que con el aumento de la cantidad de hidrógeno, las velocidades de llama laminar de metano, etano y propano aumentan linealmente aproximadamente. Además, a medida que aumenta el hidrógeno, la velocidad de la llama del metano tiene la máxima amplitud creciente entre ellos, lo que indica que el metano es más sensible a la adición de hidrógeno en la velocidad de la llama que los otros dos combustibles. Por lo tanto, la adición de hidrógeno tiene un mayor efecto en la velocidad de la llama del metano que en la del etano y el propano (Hong, Lin, y Zhu, 2016).

El objetivo del presente trabajo de investigación ha sido determinar la velocidad de la llama del Gas Natural Seco (Gas de Camisea-Perú) mediante un estudio experimental. La metodología empleada se ha soportado a través de diferentes revisiones bibliográficas de trabajos de investigación anteriores, se ha implementado un banco de ensayo apropiado y mejorado para la parte experimental considerando instrumentación adecuada y calibrada, para una comparación teórica se ha implementado los cálculos matemáticos utilizando el método de la teoría térmica y simulando en un mecanismo cinético actual utilizando el programa CHEMKIN (B, luego se ha analizado los resultados de la parte experimental comparándolos con los resultados de los cálculos matemáticos y con los resultados del programa CHEMKIN ${ }^{\circledR}$. Se han conseguido buenos resultados que han sido comparados con estudios de otros autores.

\section{MATERIALES Y MÉTODOS}

Se realiza una descripción de la sustancia portadora de energía que interviene en el proceso, detalle de las ecuaciones, equipos e instrumentos y luego se presenta la metodología para determinar la velocidad de llama.

\section{Sustancia portadora de energía}

Para este trabajo se ha utilizado el aire a condiciones ambientales del laboratorio $15^{\circ} \mathrm{C}, 82 \%$ de humedad relativa y $100.56 \mathrm{kPa}$ y gas natural seco de Camisea cuyos componentes y porcentajes se pueden encontrar en la Tabla 1. 
Tabla 1: Composición de gas natural seco de Camisea (Osinergmin, n.d.)

\begin{tabular}{|l|l|l|}
\hline Componente & Fórmula & $\%$ \\
\hline Metano & $\mathrm{CH}_{4}$ & 88.54 \\
\hline Etano & $\mathrm{C}_{2} \mathrm{H}_{6}$ & 10.32 \\
\hline Propano & $\mathrm{C}_{3} \mathrm{H}_{8}$ & 0.02 \\
\hline Iso-butano & $\mathrm{C}_{4} \mathrm{H}_{10}$ & 0.00 \\
\hline n-butano & $\mathrm{C}_{4} \mathrm{H}_{10}$ & 0.00 \\
\hline Nitrógeno & $\mathrm{N}_{2}$ & 0.54 \\
\hline Dióxido de carbono & $\mathrm{CO}_{2}$ & 0.58 \\
\hline Agua & $\mathrm{H}_{2} \mathrm{O}$ & 0.00 \\
\hline
\end{tabular}

\section{Método experimental}

Se detallan los instrumentos a utilizar en el sistema de suministro del gas natural seco de Camisea y en la Figura 1 se puede observar un esquema del sistema gas. El quemador de llama plana (Glassman et al., 2014) que se utilizó para el desarrollo del experimento fue el implementado en el laboratorio de investigación de combustión y control de emisiones gaseosas, fue fabricado en bronce y acero inoxidable, y cálculos basados en leyes físicas y empíricas. El aire suministrado se obtiene de un compresor, para la medición de datos se tiene un sensor controlador electrónico de temperatura, presión y caudal (Figura 2).

1 Tanque de gas

2 Válvula de corte

3 Regulador de presión

4 Manómetro tipo Bourdon

5 Sensor de Presión, temperatura y caudal

6 Válvula tipo aguja

7 Quemador de llama plana
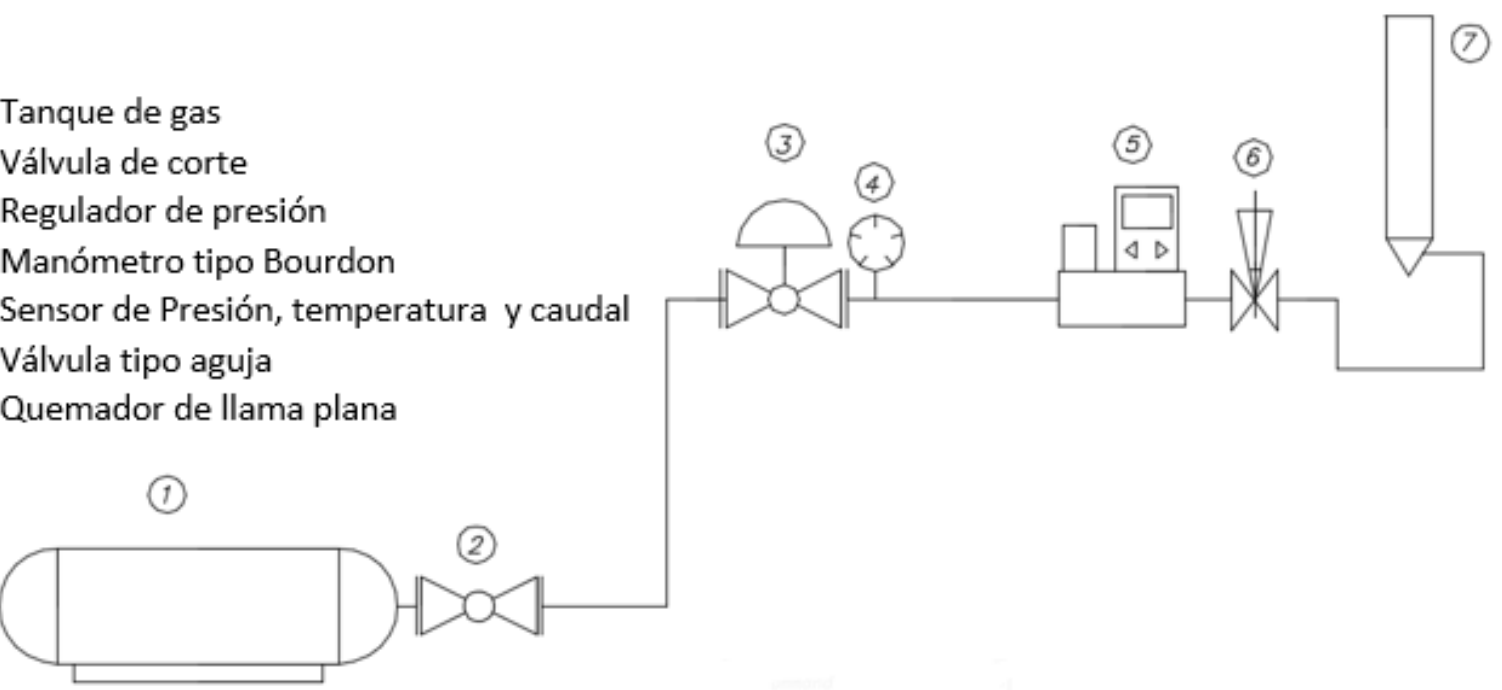

Fig. 1: Esquema de sistema de suministro de gas natural seco de Camisea

1 Compresor de aire

2 Válvula de corte

3 Válvula aguja

4 Sensor de Presión, Temperatura y caudal

5 Válvula de corte

6 Quemador de llama plana

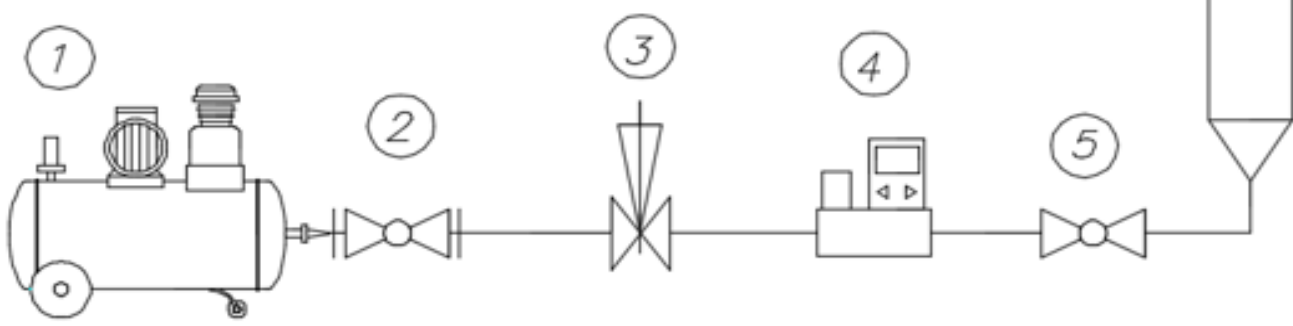

Fig. 2: Esquema de sistema de suministro de aire 
Este quemador está conformado por 3 partes principales, los cuales son el cono de admisión, el tubo mezclador (Figura 5, lado derecho) de acero inoxidable de $1 \mathrm{~m}$ de longitud y la boquilla del quemador (Figura 3 y 4), además poseen ciertos componentes complementarios como el tubo pírex (Figura 6) y su base donde reposa. En la boquilla del quemador se encuentra las piezas de cerámica refractarias los cuales son los causantes de que este perfil parabólico saliente del tubo no afecte en el perfil de la llama, debido a que las piezas de cerámicas han sido perforadas con dimensiones de $1.25 \mathrm{~mm}$ de diámetro y separación de $2 \mathrm{~mm}$ entre ellos, y la mezcla pasa por estos orificios que ocasionan una pérdida de presión uniforme. El montaje será de 2 piezas de cerámica de grosor de $12 \mathrm{~mm}$, de forma circular con diámetro de $58 \mathrm{~mm}$, ambas piezas estarán concéntricas y se asegura que todos los orificios perforados estén alineados para que exista un buen pase del flujo de la mezcla, todo esto sobre una base de acero inoxidable de la misma forma circular, donde reposan las 2 piezas y que va roscado a la parte superior del tubo de mezcla. La pieza de cerámica y la porta cerámica se pueden observar en la Figura 3 y Figura 4.

Para la determinación experimental se ha realizado algunos cálculos relacionados a los caudales, densidades del gas y el aire, con la finalidad de poder obtener los valores finales de caudal y densidad de la mezcla para luego a partir de estos valores despejar la velocidad de llama. Para poder calcular los parámetros mencionados anteriormente, se necesita tener en cuenta ciertas condiciones como presión y temperatura, estos datos aparecen en la Tabla 2. La presión y temperatura son tomados tanto como para el gas natural seco como para el aire teniendo en consideración los diferentes caudales de cada uno de ellos.

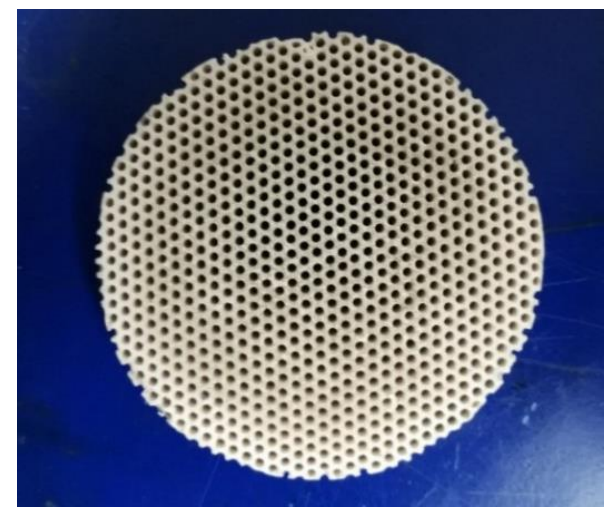

Fig. 3: Placa cerámica perforada infrarroja tipo Schwank

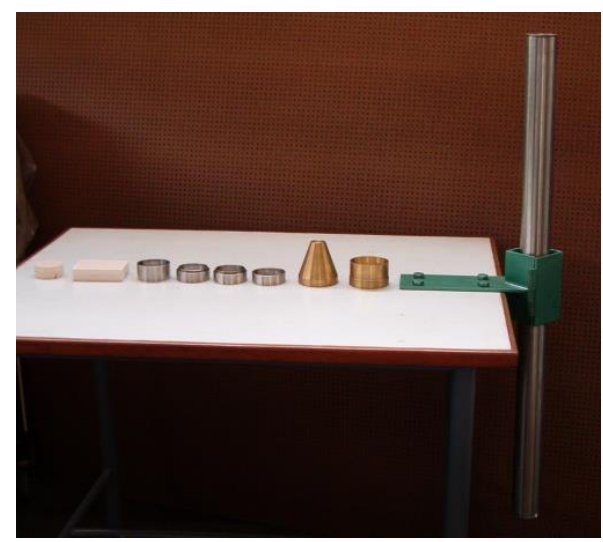

Fig. 5: Componente del quemador llama laminar

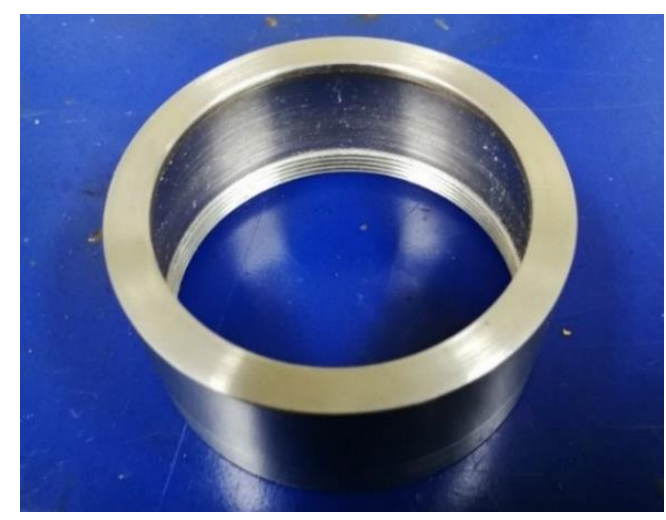

Fig. 4: Porta cerámica

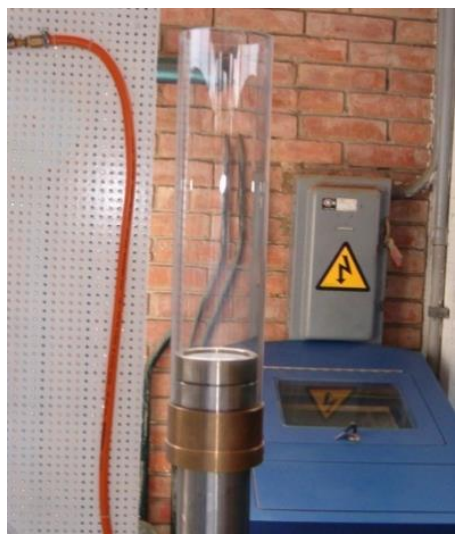

Fig. 6: Montaje del mezclador, porta cerámica y tubo pirex para estabilizar la llama

Se requiere calcular el flujo másico que entra al tubo mezclador, definido como se muestra:

$\dot{\mathrm{m}}_{\text {gas }}=\mathrm{Q}_{\text {gas }}{ }^{*} \rho_{\text {gas }}$

Donde, $\dot{\mathrm{m}}_{\mathrm{gas}}$ es el flujo másico del gas, $\mathrm{kg} / \mathrm{s}$; Qgas es el caudal del gas, $\mathrm{m}^{3} / \mathrm{s}$ y $\rho_{\text {gas }}$ es la densidad del gas, $\mathrm{m}^{3} / \mathrm{kg}$. Entonces un dato importante el cual mediremos directamente gracias al sensor de caudal descrito previamente y referenciado en la Figura 1 e ítem 5, las unidades obtenidas por el instrumento son en L/min. Mientras que, para la densidad del gas no lo obtenemos de forma tan directa, pero la podemos obtener de la ecuación de estado para los gases ideales, despejando el valor de la densidad queda expresada como:

$$
\rho_{\text {gas }}=\frac{\mathrm{P}_{\text {gas }}{ }^{*} \mathrm{M}_{\text {gas }}}{\mathrm{R}^{*} \mathrm{~T}_{\text {gas }}}
$$


Donde, $\mathrm{P}_{\text {gas }}$ es la presión absoluta del gas, $\mathrm{kPa} ; \mathrm{M}_{\text {gas }}$ es la masa molecular del gas, $\mathrm{kg} / \mathrm{kmol}$; R es la constante universal, $\mathrm{kJ} / \mathrm{kmol} . \mathrm{K}$ y $\mathrm{T}_{\text {gas }}$ es la temperatura absoluta del gas, $\mathrm{K}$. La unidad de la densidad, será de $\mathrm{g} / \mathrm{L}$ para fines de los cálculos, siendo las condiciones de entrada la presión y temperatura. Los parámetros del aire son similares a los requeridos para el gas natural seco, estos se muestran a continuación:

$$
\begin{aligned}
& \dot{\mathrm{m}}_{\text {aire }}=\mathrm{Q}_{\text {aire }}{ }^{*} \rho_{\text {aire }} \\
& \rho_{\text {aire }}=\frac{\mathrm{P}_{\text {aire }}{ }^{*} \mathrm{M}_{\text {aire }}}{\mathrm{R}^{*} \mathrm{~T}_{\text {aire }}}
\end{aligned}
$$

Donde, $\dot{\mathrm{m}}_{\text {aire }}$ es el flujo másico del aire, $\mathrm{kg} / \mathrm{s}$; Qaire es el caudal del aire, $\mathrm{m}^{3} / \mathrm{s}$; $\rho_{\text {aire }}$ es la densidad del aire, $\mathrm{m}^{3} / \mathrm{kg}$; Paire es la presión absoluta del aire, $\mathrm{kPa}$; Maire es la masa molecular del aire, $\mathrm{kg} / \mathrm{kmol}$ y Taire es la temperatura absoluta del aire, K. Las presiones y temperaturas varían debido a que el controlador de flujo de aire (Whisper-series mass flow controller, marca Alicat, hasta 250 LPM, rango de control en estado estacionario: $0.01 \%-100 \%$ ) ajusta de forma automática el flujo necesario según la relación de airecombustible que se desee por lo que el compresor trabaja de igual forma, además las unidades serán de L/min, y la densidad depende de estas pequeñas variaciones de presión y temperatura. Los parámetros de mezcla aire-combustible son los obtenidos de los diferentes datos de caudal, densidad, flujo másico del gas natural y aire, los datos finales de la mezcla se pueden obtener a partir de la conservación de masa:

$$
\dot{\mathrm{m}}_{\text {mezcla }}=\dot{\mathrm{m}}_{\text {gas }}+\dot{\mathrm{m}}_{\text {aire }}
$$

Donde, $\dot{\mathrm{m}}_{\text {mezcla }}$ es el flujo másico de la mezcla, $\mathrm{kg} / \mathrm{s}$; Qmezcla es el caudal de la mezcla, $\mathrm{m}^{3} / \mathrm{s}$ y $\rho_{\text {mezcla }}$ es la densidad de la mezcla, $\mathrm{m}^{3} / \mathrm{kg}$. Además, el flujo de masa de la mezcla puede ser expresada:

$$
\dot{\mathrm{m}}_{\text {mezcla }}=\mathrm{Q}_{\text {mezcla }}{ }^{*} \rho_{\text {mezcla }}
$$

Para poder calcular la densidad de la mezcla, recurrimos a calcular la masa molecular de la mezcla, la cual se obtiene:

$$
\mathrm{M}_{\text {mezcla }}=\sum \frac{1}{\mathrm{Xi} / \mathrm{Mi}}
$$

Donde, Xi simboliza la fracción molar de cada gas y Mi indica la masa molar para cada gas correspondiente (aire o gas natural seco), kg/kmol; como se muestra a continuación:

$$
\mathrm{Xi}=\frac{\dot{\mathrm{V}} \mathrm{i}}{\dot{\mathrm{V}}_{\text {mezcla }}}
$$

Y de la ecuación de estado podemos determinar la densidad, expresada como (Moran et al, 2004):

$$
\rho_{\text {mezcla }}=\frac{P_{\text {atm }}{ }^{*} M_{\text {mezcla }}}{\mathrm{R}^{*} T_{\text {atm }}}
$$

Donde, $\mathrm{P}_{\mathrm{atm}}$ es la presión atmosférica, $\mathrm{kPa}$ y $\mathrm{T}_{\text {atm }}$ es la temperatura absoluta a condiciones ambientales, $\mathrm{K}$. De esta forma podemos despejar el valor de caudal de la mezcla, con el que saldrá por la boquilla del quemador, y con el área obtenida de la llama, podremos obtener la velocidad de la llama, como se muestra a continuación:

$$
\mathrm{Sl}=\frac{\mathrm{Q}_{\text {mezcla }}}{\mathrm{A}_{\text {llama }}}
$$

Donde, $\mathrm{S}_{ı}$ es la velocidad de la llama, $\mathrm{m} / \mathrm{s}$ y Allama es el área de la llama, $\mathrm{m}^{2}$. La razón de equivalencia el cual muestra la relación de aire combustible comparándola con la relación ideal, se define tal como muestra a continuación:

$$
\begin{aligned}
& \mathrm{R}[\mathrm{a} / \mathrm{c}]=\frac{\dot{\mathrm{m}}_{\text {aire }}}{\dot{\mathrm{m}}_{\text {gas }}} \\
& \varnothing=\frac{\mathrm{R}[\mathrm{a} / \mathrm{c}]_{\mathrm{i}}}{\mathrm{R}[\mathrm{a} / \mathrm{c}]}
\end{aligned}
$$


Para el trabajo se tomó como referencia el valor de relación aire-combustible ideal, obteniéndose un valor de $15.52 \mathrm{~m} / \mathrm{s}$ para el gas natural seco. El área de la llama plana fue de un diámetro de $5.4 \mathrm{~cm}$, es decir un área de $22.90 \mathrm{~cm}^{2}$, sin embargo, no siempre se pudo obtener el perfil de llama plana por completo por lo que en algunos experimentos se obtuvo llama con perfil cónico o en otros casos con forma de tronco de cono, por lo que el área variará y se tomó las siguientes ecuaciones para el cálculo de su área. Cuando se obtuvo el perfil de llama plana como se muestra en la Figura 5, para el valor del área de llama se utiliza:

$$
\mathrm{A}_{\text {llama-p }}=\frac{\pi^{*} \mathrm{D}^{4}}{2}
$$

Donde, Allama-p es el área de la llama de perfil plano, $\mathrm{cm}^{2}$; D será el diámetro de la boquilla del quemador es decir $5.4 \mathrm{~cm}$, y el área será prácticamente una constante de $22.90 \mathrm{~cm}^{2}$. Si el perfil de la llama se forma cónico como se muestra en la Figura 6, el área de la llama se aproximará a la definición del área de un cono determinada por la siguiente expresión:

$$
A_{\text {llama-c }}=\pi R \sqrt{R^{2}+h^{2}}
$$

Donde, Allama-c es el área de la llama de perfil cónico, $\mathrm{cm}^{2}$; $\mathrm{R}$ es el radio de la boquilla del quemador es decir $2.7 \mathrm{~cm}$, y $\mathrm{h}$ es la altura que llega a tomar la punta del perfil de cono, cm (Balusamy et al., 2014). El último caso será cuando el perfil de la llama se forme de perfil de tronco de cono, esto debido a que el flujo que pasa por la boquilla se irá distorsionando mientras más se aleje de la boquilla debido a que el visor del vidrio de pirex posee un diámetro mayor, y el flujo de la mezcla no será uniforme en los bordes de las paredes, expresada en:

$$
A_{\text {llama-tc }}=\sqrt{\mathrm{h}^{2}+\left(\mathrm{R}^{2}-\mathrm{r}^{2}\right)^{2}} * \pi\left(\sqrt{\mathrm{h}^{2}+\left(\mathrm{R}^{2}-\mathrm{r}^{2}\right)^{2}}(\mathrm{R}+\mathrm{r})+\mathrm{R}^{2}+\mathrm{r}^{2}\right)
$$

Donde $A_{l l a m a-t c}$ es el área de la llama de perfil tronco de cono, $r$ viene a ser el radio de la base menor la cual es la que se encuentra del lado más cercano a la boquilla. En la Figura 7 se puede observar el perfil de tronco de cono.

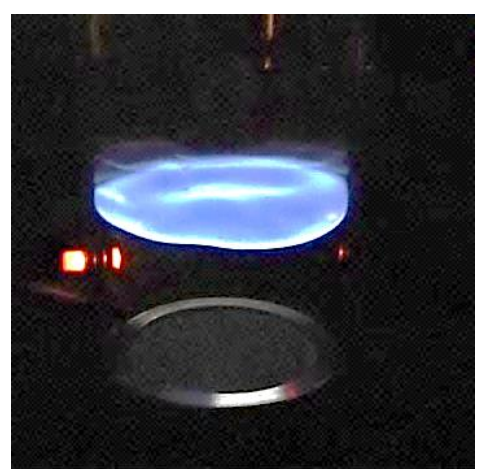

Fig. 5: Llama de perfil plano

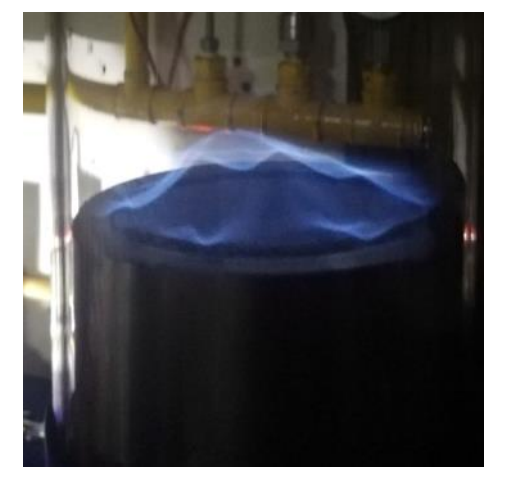

Fig. 6: Llama de perfil cónico

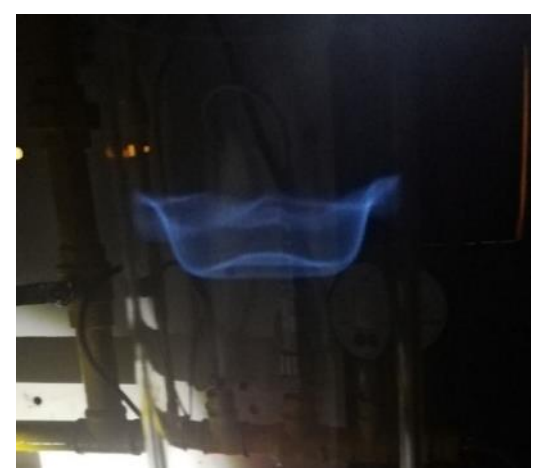

Fig.7: Llama de perfil tronco de cono

\section{Procedimiento para el experimento}

Se procede con los siguientes pasos para realizar el ensayo: 1) se verifica que todas las válvulas de corte estén cerradas, tanto para flujo de aire y gas natural seco; 2) se verifica que todas las conexiones de mangueras estén correctamente ajustadas con las abrazaderas, luego se realiza una prueba de hermeticidad para evitar cualquier fuga de aire o combustible; 3) se energiza todo el panel de control, así como los sistemas de suministro del aire y gas natural seco para la lectura de los datos en el registrador electrónico; 4) se regula la presión de salida del aire en el mismo compresor, a un valor de 1 bar para tener aire comprimido en el tanque pulmón; 5) se regula la presión del gas y se ajusta aproximadamente a los 20 mbar; 6) se abre la válvula del sistema de gas y se enciende la llama a través de una chispa en la boquilla del quemador originándose la combustión inicialmente con tan solo el aire del medio ambiente; 7) se regula un caudal definido a trabajar; 8) se deja pasar el aire del compresor hacia el sistema; 9) se espera un momento hasta que se estabilice el flujo de la mezcla para poder insertar el visor tubo de vidrio pírex (para darle estabilidad por posibles perturbaciones del medio); 10) se va regulando el aire hasta obtener una llama plana, o en caso no poder obtener una llama plana, será hasta obtener una llama estable; y 11) una vez obtenida la llama plana o estabilizada, se toma los datos de presión temperatura y caudal tanto de aire y gas natural seco. El procedimiento para la parte experimental se realizó en base a los diferentes trabajos de la literatura y a la experiencia del autor. 


\section{RESULTADOS Y DISCUSIÓN}

Se presenta una selección de 28 datos experimentales de los datos medidos, calculados de mezcla airecombustible y de velocidad de llama en función de la razón de equivalencia. Los datos medidos se muestran en la Tabla 2 principalmente de caudal, presión y temperatura del gas natural seco y del aire. La toma de datos se realiza mediante instrumentación apropiada que pueda registrar en tiempo real y ser grabado cada segundo.

Tabla 2: Datos medidos de gas natural seco y aire

\begin{tabular}{|l|l|l|l|l|l|l|}
\hline \multirow{2}{*}{$N^{\circ}$ exp } & \multicolumn{3}{|l|}{ Gas natural seco } & \multicolumn{4}{l|}{ Aire } \\
\hline 1 & $\mathrm{Q}_{\mathrm{gn}}\left(\mathrm{m}^{3} / \mathrm{min}\right)$ & $\mathrm{p}_{\mathrm{gn}}(\mathrm{kPa})$ & $\mathrm{T}_{\mathrm{gn}}(\mathrm{K})$ & $\mathrm{Q}_{\mathrm{a}}\left(\mathrm{m}^{3} / \mathrm{min}\right)$ & $\mathrm{p}_{\mathrm{a}}(\mathrm{kPa})$ & $\mathrm{T}_{\mathrm{a}}(\mathrm{K})$ \\
\hline 2 & 0.00101 & 1.0234 & 298.33 & 0.0176 & 0.9993 & 298.33 \\
\hline 3 & 0.00122 & 1.0221 & 296.21 & 0.0225 & 0.9993 & 296.29 \\
\hline 4 & 0.00099 & 1.0221 & 296.96 & 0.0185 & 0.9966 & 297.62 \\
\hline 5 & 0.00100 & 1.0234 & 298.53 & 0.0174 & 0.9966 & 298.01 \\
\hline 6 & 0.00106 & 1.0234 & 299.01 & 0.0177 & 0.9931 & 301.63 \\
\hline 7 & 0.00094 & 1.0234 & 299.05 & 0.0187 & 0.9931 & 301.63 \\
\hline 8 & 0.00095 & 1.0241 & 299.11 & 0.0179 & 0.9966 & 299.73 \\
\hline 9 & 0.00105 & 1.0324 & 295.65 & 0.0133 & 0.9966 & 299.90 \\
\hline 10 & 0.00140 & 1.0324 & 296.36 & 0.0160 & 0.9979 & 294.00 \\
\hline 11 & 0.00070 & 1.0303 & 294.02 & 0.0100 & 0.9924 & 292.60 \\
\hline 12 & 0.00053 & 1.0317 & 294.16 & 0.0084 & 0.9917 & 292.77 \\
\hline 13 & 0.00116 & 1.0297 & 294.51 & 0.0141 & 0.9938 & 293.30 \\
\hline 14 & 0.00090 & 1.0297 & 294.51 & 0.0118 & 0.9917 & 293.10 \\
\hline 15 & 0.00057 & 1.0276 & 300.66 & 0.0087 & 0.9938 & 297.56 \\
\hline 16 & 0.00059 & 1.0276 & 300.66 & 0.0088 & 0.9938 & 297.88 \\
\hline 17 & 0.00041 & 1.0283 & 300.66 & 0.0066 & 0.9931 & 298.33 \\
\hline 18 & 0.00078 & 1.0283 & 300.13 & 0.0113 & 0.9945 & 298.79 \\
\hline 19 & 0.00108 & 1.0269 & 300.09 & 0.0071 & 0.9938 & 301.24 \\
\hline 20 & 0.00198 & 1.0248 & 300.84 & 0.0154 & 0.9966 & 301.24 \\
\hline 21 & 0.00199 & 1.0248 & 300.84 & 0.0154 & 0.9966 & 301.24 \\
\hline 22 & 0.00199 & 1.0248 & 300.84 & 0.0199 & 0.9966 & 301.24 \\
\hline 23 & 0.00145 & 1.0255 & 293.09 & 0.0100 & 1.0000 & 291.30 \\
\hline 24 & 0.00209 & 1.0234 & 293.52 & 0.0153 & 1.0014 & 291.77 \\
\hline 25 & 0.00270 & 1.0297 & 293.69 & 0.0202 & 1.0055 & 292.01 \\
\hline 26 & 0.00324 & 1.0276 & 293.86 & 0.0250 & 1.0062 & 287.59 \\
\hline 27 & 0.00373 & 1.0269 & 294.12 & 0.0299 & 1.0103 & 292.55 \\
\hline 28 & 0.00478 & 1.0255 & 294.37 & 0.0397 & 1.0138 & 292.27 \\
\hline & & & & & & \\
\hline
\end{tabular}

La Tabla 3 muestra los resultados calculados mediante el modelo de mezcla de gases ideales. Siendo $r \mathrm{ro}_{m}$ la densidad de la mezcla, $m_{m}$ el flujo másico, $Q_{m}$ el caudal.

Tabla 3: Cálculos de la mezcla de gases

\begin{tabular}{|l|l|l|l|l|l|}
\hline $\mathrm{N}^{\circ} \exp$ & $\mathrm{m}_{\mathrm{m}}(\mathrm{g} / \mathrm{s})$ & $\mathrm{M}_{\mathrm{m}}$ & hho $_{\mathrm{m}}(\mathrm{g} / \mathrm{L})$ & $\mathrm{Q}_{\mathrm{m}}(\mathrm{L} / \mathrm{min})$ & $\mathrm{Q}_{\mathrm{m}}\left(\mathrm{m}^{3} / \mathrm{min}\right)$ \\
\hline 1 & 0.35459 & 28.280 & 1.176 & 18.09 & 0.01809 \\
\hline 2 & 0.45554 & 28.313 & 1.178 & 23.21 & 0.02321 \\
\hline 3 & 0.37187 & 28.312 & 1.178 & 18.95 & 0.01895 \\
\hline 4 & 0.34988 & 28.284 & 1.177 & 17.84 & 0.01784 \\
\hline 5 & 0.35050 & 28.280 & 1.176 & 17.88 & 0.01788 \\
\hline 6 & 0.37036 & 28.278 & 1.176 & 18.89 & 0.01889 \\
\hline 7 & 0.34924 & 28.315 & 1.178 & 17.79 & 0.01779 \\
\hline 8 & 0.35689 & 28.321 & 1.178 & 18.18 & 0.01818 \\
\hline 9 & 0.27476 & 28.059 & 1.167 & 14.12 & 0.01412 \\
\hline 10 & 0.33182 & 27.977 & 1.164 & 17.11 & 0.01711 \\
\hline 11 & 0.20563 & 28.146 & 1.171 & 10.54 & 0.01054 \\
\hline 12 & 0.17180 & 28.215 & 1.174 & 8.78 & 0.00878 \\
\hline 13 & 0.29178 & 28.026 & 1.166 & 15.02 & 0.01502 \\
\hline
\end{tabular}


Tabla 3: continuación

\begin{tabular}{|l|l|l|l|l|l|}
\hline $\mathrm{N}^{\circ} \exp$ & $\mathrm{m}_{\mathrm{m}}(\mathrm{g} / \mathrm{s})$ & $\mathrm{M}_{\mathrm{m}}$ & $\mathrm{rho}_{\mathrm{m}}(\mathrm{g} / \mathrm{L})$ & $\mathrm{Q}_{\mathrm{m}}(\mathrm{L} / \mathrm{min})$ & $\mathrm{Q}_{\mathrm{m}}\left(\mathrm{m}^{3} / \mathrm{min}\right)$ \\
\hline 14 & 0.24298 & 28.084 & 1.168 & 12.48 & 0.01248 \\
\hline 15 & 0.17562 & 28.198 & 1.173 & 8.98 & 0.00898 \\
\hline 16 & 0.17761 & 28.182 & 1.172 & 9.09 & 0.00909 \\
\hline 17 & 0.13254 & 28.230 & 1.174 & 6.77 & 0.00677 \\
\hline 18 & 0.22785 & 28.159 & 1.171 & 11.67 & 0.01167 \\
\hline 19 & 0.14911 & 27.434 & 1.122 & 7.97 & 0.00797 \\
\hline 20 & 0.31973 & 27.652 & 1.131 & 16.96 & 0.01696 \\
\hline 21 & 0.31985 & 27.646 & 1.131 & 16.97 & 0.01697 \\
\hline 22 & 0.40667 & 27.919 & 1.142 & 21.37 & 0.02137 \\
\hline 23 & 0.21732 & 27.519 & 1.125 & 11.59 & 0.01159 \\
\hline 24 & 0.33074 & 27.597 & 1.129 & 17.58 & 0.01758 \\
\hline 25 & 0.43744 & 27.620 & 1.130 & 23.23 & 0.02323 \\
\hline 26 & 0.54805 & 27.678 & 1.132 & 29.05 & 0.02905 \\
\hline 27 & 0.64575 & 27.708 & 1.133 & 34.19 & 0.03419 \\
\hline 28 & 0.85862 & 27.754 & 1.135 & 45.39 & 0.04539 \\
\hline
\end{tabular}

En la Tabla 4 se muestran los datos encontrados experimentalmente de velocidad de llama ( $V_{I I)}$ según la razón de equivalencia (phi). Siendo $R C A$ la relación combustible-aire.

Tabla 4: Resultado de velocidad de llama del Gas Natural seco de Camisea

\begin{tabular}{|c|c|c|c|}
\hline$N^{\circ} \exp$ & $\mathrm{V}_{\text {II }}(\mathrm{cm} / \mathrm{s})$ & RCA & phi \\
\hline 1 & 7.40 & 27.85 & 0.59 \\
\hline 2 & 9.50 & 29.51 & 0.56 \\
\hline 3 & 7.76 & 29.46 & 0.56 \\
\hline 4 & 7.30 & 28.06 & 0.59 \\
\hline 5 & 7.32 & 27.87 & 0.59 \\
\hline 6 & 7.73 & 27.75 & 0.60 \\
\hline 7 & 7.28 & 29.60 & 0.56 \\
\hline 8 & 7.44 & 29.93 & 0.55 \\
\hline 9 & 27.68 & 20.12 & 0.82 \\
\hline 10 & 33.53 & 18.18 & 0.91 \\
\hline 11 & 20.65 & 22.63 & 0.73 \\
\hline 12 & 17.21 & 25.05 & 0.66 \\
\hline 13 & 29.43 & 19.28 & 0.86 \\
\hline 14 & 24.46 & 20.77 & 0.80 \\
\hline 15 & 17.60 & 24.41 & 0.68 \\
\hline 16 & 17.82 & 23.83 & 0.69 \\
\hline 17 & 13.27 & 25.64 & 0.64 \\
\hline 18 & 22.87 & 23.04 & 0.72 \\
\hline 19 & 9.01 & 10.37 & 1.59 \\
\hline 20 & 15.59 & 12.36 & 1.34 \\
\hline 21 & 15.61 & 12.30 & 1.34 \\
\hline 22 & 22.50 & 15.91 & 1.04 \\
\hline 23 & 11.67 & 11.07 & 1.49 \\
\hline 24 & 14.56 & 11.79 & 1.40 \\
\hline 25 & 17.79 & 12.03 & 1.37 \\
\hline 26 & 20.40 & 12.64 & 1.31 \\
\hline 27 & 21.81 & 12.98 & 1.27 \\
\hline 28 & 27.48 & 13.53 & 1.22 \\
\hline
\end{tabular}

En la Tabla 5 se muestran la temperatura adiabática calculada analíticamente y el calculado mediante el software científico CHEMKIN ® (Los mecanismos usados en Chemkin® son el Gri-Mech, más utilizado para simular gas natural y Aramvo Meche, considerando presión y temperatura según la Tabla 2 para el gas natural y aire. Además, se consideraron como entrada las especies del gas natural de Camisea. 
Tabla 5: Temperatura Adiabática del Gas Natural Seco de Camisea

\begin{tabular}{|l|l|l|l|}
\hline Tipo de mezcla & $\Phi$ & Temperatura adiabática Calculado (K) & Temperatura adiabática Chemkin (K) \\
\hline \multirow{5}{*}{ Mezcla Pobre } & 0.5 & 1484 & 1482.1 \\
\cline { 2 - 5 } & 0.6 & 1671 & 1668.6 \\
\cline { 2 - 5 } & 0.7 & 1851 & 1841.9 \\
\cline { 2 - 4 } & 0.8 & 2021 & 2000.6 \\
\cline { 2 - 4 } Estequiométrica & 0.9 & 2183 & 2137.9 \\
\hline \multirow{5}{*}{ Mezcla Rica } & 1 & 2334 & 2228.8 \\
\hline & 1.1 & 2235 & 2215.1 \\
\cline { 2 - 4 } & 1.2 & 2132 & 2142.0 \\
\cline { 2 - 4 } & 1.3 & 2042 & 2063.0 \\
\cline { 2 - 4 } & 1.4 & 1940 & 1985.6 \\
\cline { 2 - 4 } & 1.5 & 1863 & 1910.7 \\
\cline { 2 - 4 } & 1.6 & 1756 & 1838.3 \\
\hline
\end{tabular}

Estas temperaturas sirven para calcular matemáticamente la velocidad de llama. Estas temperaturas se pueden diferenciar según se muestra en la Figura 8. En la Figura 9 se muestran los resultados de velocidad de llama según los datos calculados de la Tabla 5. Además, estas son comparadas con otros autores para gases puros (Vagelopoulos et al., 1994). Encontrándose una misma tendencia, validando el modelo matemático empleado.

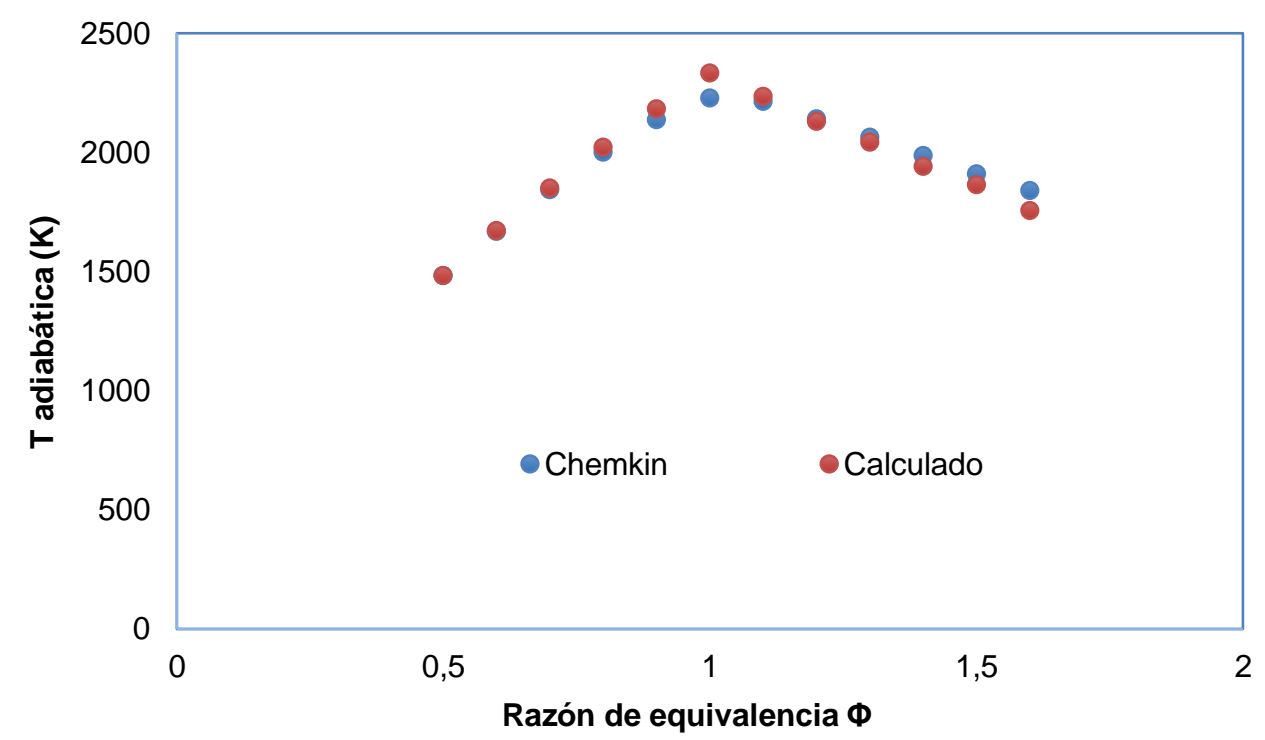

Fig.8: Temperatura de llama adiabática del gas natural calculado y según CHEMKIN ${ }^{\circledR}$ vs Razón de equivalencia

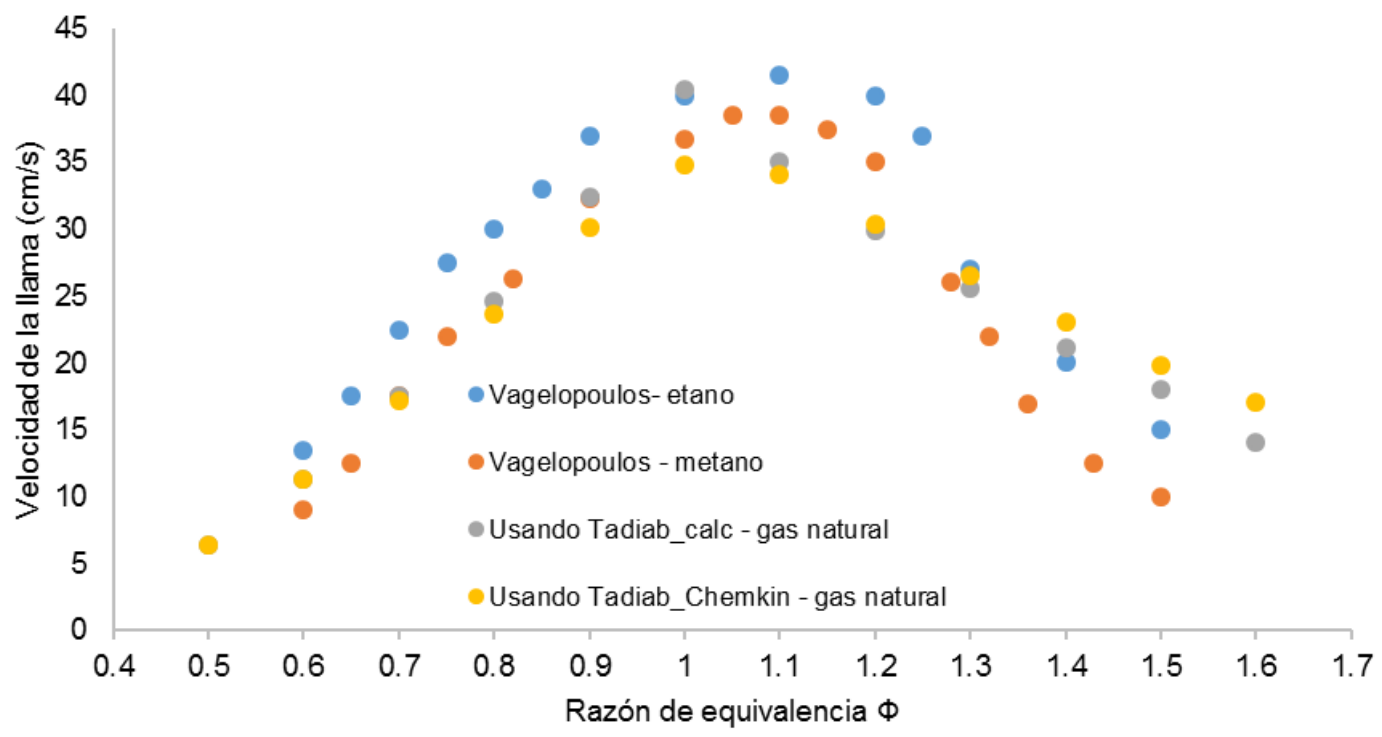

Fig. 9: Velocidad de llama del gas natural seco calculada analíticamente y según CHEMKIN ${ }^{\circledR}$ vs razón de equivalencia 
En la Figura 9 se puede discutir que en la zona de mezcla pobre para el Gas Natural Seco de Camisea la temperatura adiabática calculada analíticamente (Glassman et al., 2014) es mayor que la calculada mediante el software científico CHEMKIN ${ }^{\circledR}$ y en la zona de mezcla rica sucede todo lo contrario. La velocidad de llama obtenida es siguiendo el modelo de Mallard y Le Chatelier (Glassman et al., 2014) y la temperatura adiabática encontrada por el software CHEMKIN ${ }^{\circledR}$. También se visualiza que la velocidad de llama calculada y según CHEMKIN ${ }^{\circledR}$ son muy similares al encontrado por Vagelopoulos (1994) para el gas metano.

Para una mezcla pobre los resultados encontrados experimentalmente según la Figura 10 se encuentran entre los valores del metano y etano encontrados por Vagelopoulos en los rangos de 0.55 al 0.9 y otros autores (Vagelopoulos et al., 1994) (Gu et al., 2000). En el caso del gas natural de Camisea este tiene un porcentaje de metano del $88.54 \%$ y de etano del $10.32 \%$ por lo tanto, la curva de velocidad de llama en la parte experimental tiende a estar más cerca a lo encontrado por Vagelopoulos en metano, coincidiendo lo que prevalece como mayor porcentaje de metano en el gas natural de Camisea. Por otro lado, también es importante el tipo de quemador utilizado, que en este caso se usó el mostrado en las Figuras 5 y 6.

Al comparar con otros autores los resultados experimentales se encuentran por encima de los valores encontrados por Hassan y Van Maaren (Hassan et al., 1998) (Van Maaren et al., 1994). Los valores son consistentes y guardan la misma tendencia y similitud, pero difieren de los valores debido a que el gas natural de Camisea y otros tienen porcentajes diferentes en su composición química. También, para una mezcla pobre se coincide con lo manifestado por Dirrenberger (2011) al estar la velocidad de la llama entre el metano y el etano.

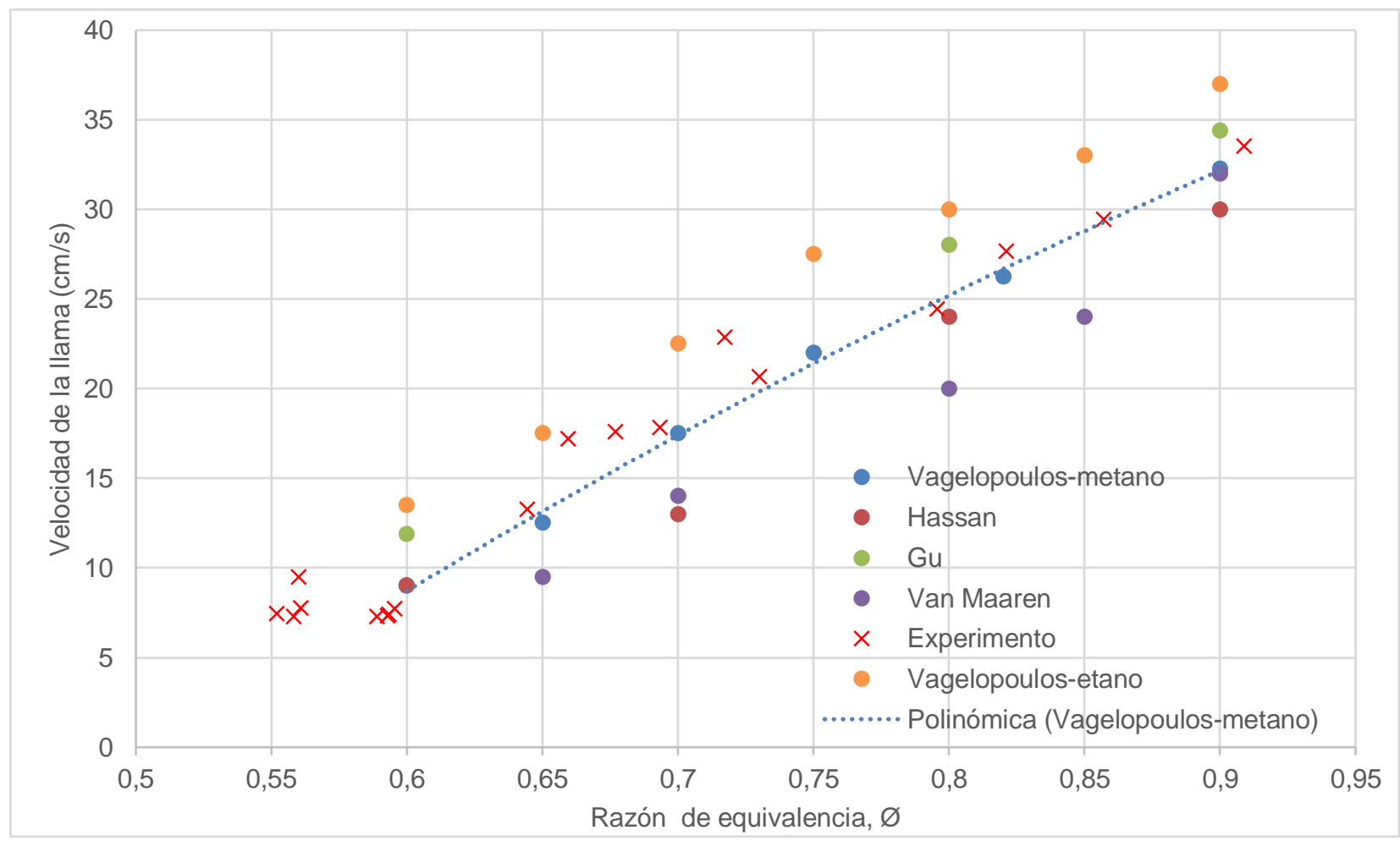

Fig. 10: Velocidad de llama del gas natural experimental en mezcla pobre

Al comparar con otros autores según se aprecia en la Figura 11 para una mezcla rica guardan una misma similitud en la tendencia más no en los valores. En el rango de razón de equivalencia de 1.2 a 1.35 los resultados encontrados son por debajo de lo que encontró Vagelopoulos en relación a gases puros como el metano y el etano, pero en el rango de 1.35 al 1.5 se encuentra entre los valores de metano y etano presentados por el mismo autor (Vagelopoulos et al., 1994). Comparándolo con otro autor los valores encontrados superan los valores entre el rango de 1.25 a 1.5 a los que encontró Van Maaren (Van Maaren et al., 1994).

Estos resultados amplios en mezcla rica y pobre se dio gracias a la incorporación en este proyecto de un sistema compresor que incluía un regulador de gas aguas arriba el cual mantenía constante la presión de gas permitiendo una adecuada mezcla aire-combustible y sin pulsaciones cuando en un anterior proyecto solo se usaba una válvula de aguja como elemento de regulación de presión. 


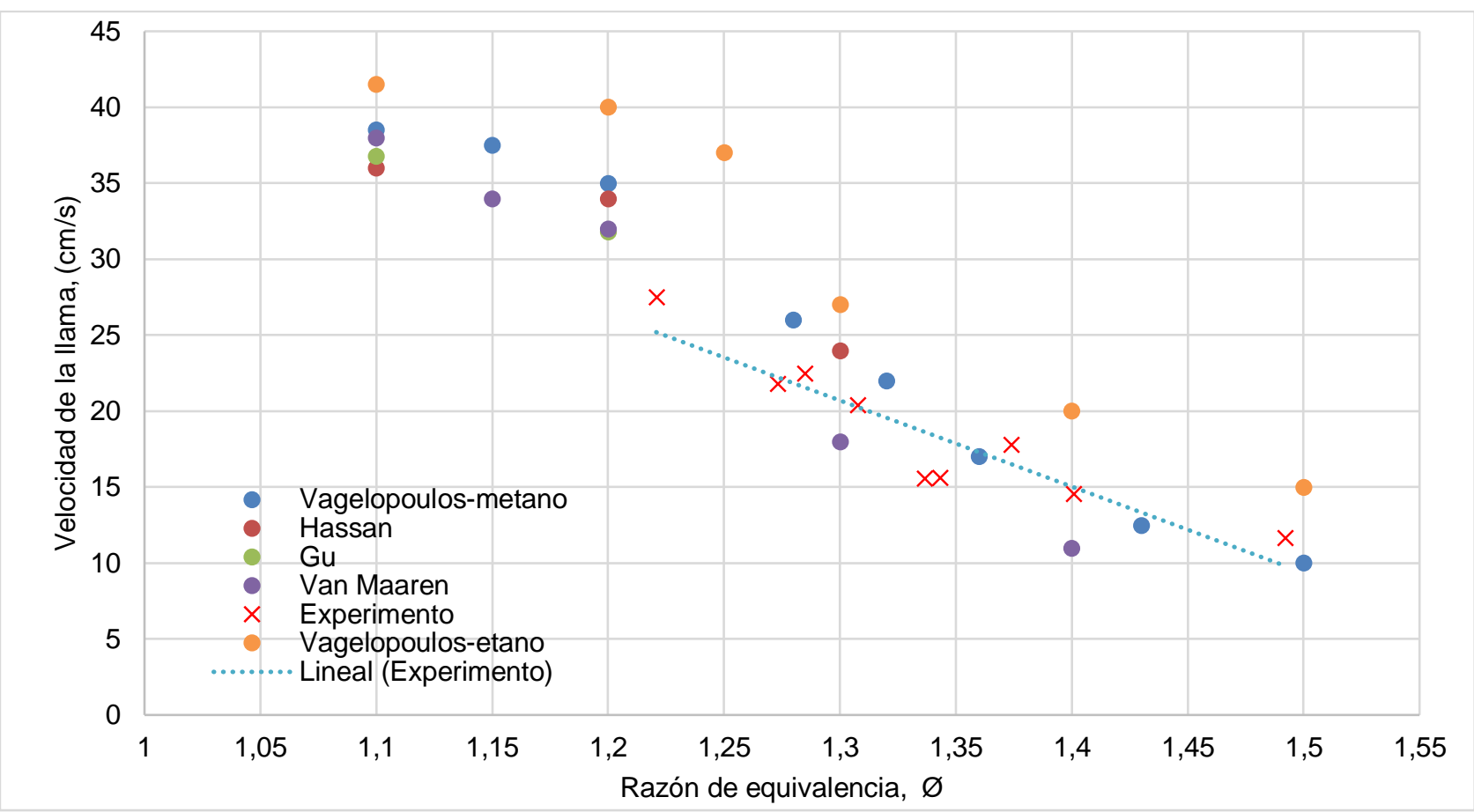

Fig. 11: Velocidad de llama del gas natural experimental en mezcla rica

\section{CONCLUSIONES}

A partir de los resultados obtenidos, se pueden extraer las siguientes conclusiones:

1) Los resultados encontrados de velocidad de llama del Gas Natural Seco de Camisea son los esperados al comparar con otros resultados por otros autores;

2) El modelo matemático empleado y usando CHEMKIN ${ }^{\circledR}$ nos muestran unos valores más próximos al gas puro metano en la zona de mezcla pobre y mayores al etano y metano en mezcla rica en el rango de 1.4 al 1.6 de razón de equivalencia y estos son muy semejantes a los resultados experimentales en este trabajo;

3) Los valores experimentales difieren de los resultados de CHEMKIN ${ }^{\circledR}$ en la zona de mezcla rica entre los valores de 1.35 al 1.5 de razón de equivalencia;

4) La implementación del quemador de llama plana y la incorporación de un tanque pulmón de aire hicieron que se obtuviera un amplio rango de velocidad de llama en mezcla rica y pobre; y

5) La metodología empleada en la parte experimental (mezclador y placa cerámica perforada infrarroja tipo schwank) nos proporciona una adecuada base de datos de velocidad de llama comparado con otros métodos discutidos en la bibliográfia, en este caso para el gas natural de Camisea-Perú.

\section{AGRADECIMIENTOS}

Se agradece a la Dirección de Gestión de la Investigación (DGI) que a través de los Proyectos Concursables de Investigación Básica y Aplicada 591-DGI-2019 de la Pontificia Universidad Católica del Perú financiaron la investigación.

\section{REFERENCIAS}

Amirante, R., Distaso, E., y otros 2 autores. Laminar flame speed correlations for methane, ethane, propane and their mixtures, and natural gas and gasoline for spark-ignition engine simulations, http://doi.org/10.1177/1468087417720018, International Journal of Engine Research, 18(9), 951-970 (2017)

Balusamy, S., Cessou, A., y Lecordier, B. Laminar propagation of lean premixed flames ignited in stratified mixture, doi: 10.1016/j.combustflame.2013.08.023, Combustion and Flame, 161(2), 427-437 (2014)

Cardona, C., Amell, A., y Burbano, H. Laminar Burning Velocity of Natural Gas / Syngas-Air Mixture. Dyna, 80(180), 136143 (2013)

Dirrenberger, P., Le Gall, H., y otros 5 autores. Measurements of laminar flame velocity for components of natural gas, http://doi.org/10.1021/ef200707h, Energy and Fuels, 25(9), 3875-3884 (2011) 
Glassman, I., Yetter, R.A., y Glumac, N. Combustion. Academic Press. (2014)

$\mathrm{Gu}, \mathrm{X}$. J., Haq, M. Z., y otros 2 autores. Laminar burning velocity and Markstein lengths of methane-air mixtures, http://doi.org/10.1016/S0010-2180(99)00142-X, Combustion and Flame, 121(1-2), 41-58 (2000)

Hassan, M. I., Aung, K. T., y Faeth, G. M. Measured and predicted properties of laminar premixed methane/air flames at various pressures, http://doi.org/10.1016/S0010-2180(98)00025-X, Combustion and Flame, 115(4), 539-550 (1998)

Hong, Y.D., Lin, B.Q., y Zhu, C.J. Premixed methane/air gas deflagration simulations in closed-end and open-end tubes, http://doi.org/10.1177/1756827716648640, International Journal of Spray and Combustion Dynamics, 8(4), 271-284 (2016)

Moran, M. J., Shapiro, H. N., y otros 2 autores. Fundamentos de Termodinámica Técnica. Editorial Reverté (2004)

Osinergmin. (n.d.). Osinergmin - Gas Natural - Información general.

Plichta, D., Mathieu, O., y otros 5 autores. Laminar flame speeds of natural gas blends with hydrogen at elevated pressures and temperatures. 8th US National Combustion Meeting 2013, 3(2012) (2013)

Tamayo, J., Salvador, J., y otros 2 autores, La industria de la electricidad en el Perú: 25 años de aportes al crecimiento económico del país (2016)

Vagelopoulos, C. M., Egolfopoulos, F. N., y Law, C. K.. Further considerations on the determination of laminar flame speeds with the counterflow twin-flame technique, http://doi.org/10.1016/S0082-0784(06)80776-9, Symposium (International) on Combustion, 25(1), 1341-1347 (1994)

Van Maaren, A., Thung, D. S., y De Goey, L.P.H. Measurement of Flame Temperature and Adiabatic Burning Velocity of Methane/Air Mixtures, http://doi.org/10.1080/00102209408935360, Combustion Science and Technology, 96(4-6), 327344 (1994)

Zhang, B., Dong, C., y otros 5 autores. Experimental study on laminar flame speed of natural gas/carbon monoxide/air mixtures, http://doi.org/10.1080/15567036.2011.588681, Energy Sources, Part A: Recovery, Utilization and Environmental Effects, 37(6), 576-582 (2015) 
